\title{
STANDARD-LIKE MODELS FROM D-BRANES
}

\author{
D. BAILIN \\ Centre for Theoretical Physics \\ University of Sussex \\ Brighton, BN1 9QJ, UK \\ E-mail:D.Bailin@sussex.ac.uk
}

\begin{abstract}
I describe the main features of new intersecting D4- and D5-brane orbifold models that yield the non-supersymmetric standard model, up to vector-like matter and, in some cases, extra $U(1)$ factors in the gauge group. There are six-stack D4-brane models that have charged-singlet scalar tachyons and which either contain all of the Yukawa couplings to the tachyonic Higgs doublets that are needed to generate mass terms for the fermions at renormalisable level or possess an unwanted extra $U(1)$ gauge symmetry after spontaneous symmetry breaking. In the D5-brane models a minimum of eight stacks is needed.
\end{abstract}

\section{Introduction}

The D-brane world offers an attractive, bottom-up route to getting standard-like models from Type II string theory ${ }^{1}$. Open strings that begin and end on a stack $a$ of $N_{a}$ D-branes generate the gauge bosons of the group $U\left(N_{a}\right)$ living in the world volume of the D-branes. So the standard approach is to start with one stack $a=1$ of three D-branes, another $a=2$ of two D-branes, and $n$ other stacks each having just one D-brane, thereby generating the gauge group $U(3) \times U(2) \times U(1)^{n}$. Fermions in bifundamental representations of the corresponding gauge groups can arise at the intersections of such stacks ${ }^{2}$, but to get $D=4$ chiral fermions the intersecting branes should sit at a singular point in the space transverse to the branes, an orbifold fixed point, for example. In general, such configurations yield a non-supersymmetric spectrum, so to avoid the hierarchy problem the string scale associated with such models must be no more than a few $\mathrm{TeV}$. Gravitational interactions occur in the bulk ten-dimensional space, and to ensure that the Planck energy has its observed large value, it is necessary that there are large dimensions transverse to the branes ${ }^{3}$. The D-branes with which we are concerned wrap the 3 -space we inhabit and closed 1-, 2- or 3-cycles of a toroidally compactified $T^{2}, T^{2} \times T^{2}$ or $T^{2} \times T^{2} \times T^{2}$ space. Thus getting the correct Planck scale effectively means that only D4- 
and D5-brane models are viable, since for D6-branes there is no dimension transverse to all of the intersecting branes.

During the past year orientifold models with intersecting D6- and D5branes have been constructed that yield precisely the fermionic spectrum of the standard model (plus three generations of right-chiral neutrinos) $)^{4,5}$. (Other recent work on intersecting brane models, both supersymmetric and non-supersymmetric, and their phenomenological implications may be found $i{ }^{6}$.) The spectrum includes $S U(2)_{L}$ doublet scalar tachyons that may be regarded as the Higgs doublets that break the electroweak symmetry group, but also, unavoidably, colour-triplet and charged singlet tachyons either of which is potentially fatal for the phenomenology. In a previous paper $^{7}$ we studied D4-brane models, transverse to a $Z_{3}$ orbifold, having three generations of chiral matter that were constrained to have no colour triplet or charged singlet scalar tachyons, and which contained the Yukawa couplings to the Higgs doublets needed to give masses to all quarks and leptons; these models, unavoidably, also possessed extra, vector-like leptonic matter. The wrapping numbers of the various stacks are constrained by the requirement of Ramond-Ramond tadpole cancellation and also by the requirement that the mixed $U(1)_{Y} \times S U(2)_{L}^{2}$ and $U(1)_{Y} \times S U(3)_{c}^{2}$ anomalies cancel. In string theory, these latter constraints derive from a generalised Green-Schwarz mechanism that ensures that the gauge bosons associated with all anomalous $U(1)$ s acquire string-scale masses ${ }^{8}$. In fact the cancellation of anomalies is necessary, but not sufficient ${ }^{4}$, to ensure the masslessness of the gauge boson associated with $U(1)_{Y}$. When the stronger constraints derived from this Green-Schwarz mechanism are applied we find that in the models constructed in ${ }^{7}$ the weak hypercharge $U(1)_{Y}$ survives only as a global, not a local, symmetry ${ }^{\mathrm{a}}$. A recent paper ${ }^{10}$ was the first to construct semi-realistic intersecting D4-brane orbifold models without this defect. In this talk I outline the features of our new D4-brane models and some results from our attempts to construct analogous models using D5-branes.

\section{D4-brane models}

The stacks of D4-branes wrap closed 1-cycles of $T^{2}$ and are all situated at a fixed point of the transverse $T^{2} \times T^{2} / Z_{3}$ orbifold. A stack $a$ is specified by two wrapping numbers $\left(n_{a}, m_{a}\right)$ that specify the number of times $a$ wraps the basis 1-cycles of $T^{2}$. The generator $\theta$ of the $Z_{3}$ point group is embedded in the stack of $N_{a}$ branes as $\gamma_{\theta, a}=\alpha^{p_{a}} I_{N_{a}}$, where $\alpha=e^{2 \pi i / 3}, p_{a}=0,1,2$.

${ }^{a}$ A similar observation applies also to the models constructed $i^{9}$ and Kataoka and Shimojo ${ }^{6}$. 
The first two stacks $a=1,2$ defined above, that generate a $U(3) \times U(2)$ gauge group, are common to all models; their Chan-Paton factors $\alpha^{p_{a}}$ have $p_{1} \neq p_{2}$. Besides these we have, in general, three sets $I, J, K$ of $U(1)$ stacks characterised by their Chan-Paton factors: $p_{i}=p_{2} \forall i \in I, p_{1} \neq p_{j} \neq$ $p_{2} \forall j \in J$, and $p_{k}=p_{1} \forall k \in K$; the sets $I, J, K$ are each divided into two subsets $I_{1} \cup I_{2}=I$ etc., defined so that the weak hypercharge $Y$ is the linear combination of the $U(1)$ charges $Q_{a}$

$$
-Y=\frac{1}{3} Q_{1}+\frac{1}{2} Q_{2}+\sum_{i_{1} \in I_{1}} Q_{i_{1}}+\sum_{j_{1} \in J_{1}} Q_{j_{1}}+\sum_{k_{1} \in K_{1}} Q_{k_{1}}
$$

In general, tachyonic scalars arise at intersections between stacks $a$ and $b$ which have the same Chan-Paton factor $p_{a}=p_{b}$; the number $I_{a b}=$ $n_{a} m_{b}-m_{a} n_{b}$ of intersections gives the number of such particles. Thus, Higgs doublets, which are needed to give mass to the fermionic matter, arise at $\left(2 i_{1}\right)$ and $\left(2 i_{2}\right)$ intersections ${ }^{\mathrm{b}}$. $\operatorname{In}^{10}$ we obtained general solutions for the wrapping numbers of three-generation models that satisfy the constraints deriving from twisted tadpole cancellation and the requirement that the $U(1)_{Y}$ gauge boson associated with weak hypercharge does not get a Green-Schwarz mass. We also demand that there are tree-level Yukawa couplings needed to give masses to all matter. As before ${ }^{7}$, the three fermion generations include right-chiral neutrinos in all models. At least six stacks are required, and there is a unique, one-parameter family of six-stack models having stacks $1,2, i_{1}, i_{2}, j_{1}, j_{2}$ that include the (tachyonic) Higgs doublets needed to generate mass terms for all matter. They have extra vector-like leptonic, but not quark, matter. We find

$$
12(L+\bar{L})+6\left(e_{L}^{c}+\bar{e}_{L}^{c}\right)+3\left(\nu_{L}^{c}+\bar{\nu}_{L}^{c}\right)
$$

Besides 9 Higgs doublets, there are also 3 charged-singlet tachyons, but no colour-triplet tachyons. The gauge coupling strengths are inversely proportional to the length of the 1-cycle wrapped by the corresponding stack, so the wrapping numbers determine gauge coupling constant ratios, at the string scale. The ratios predicted by the above six-stack models are inconsistent with those measured at the electroweak scale, but our previous experience ${ }^{7}$ suggests that they might be consistent with only a modest amount of renormalisation group running, so they are not necessarily inconsistent with a string scale of a few $\mathrm{TeV}$ that is required for

${ }^{\mathrm{b}}$ In our models, as in all others, the Higgs content is non-minimal; this seems to be a generic feature of models deriving from string theory ${ }^{11}$. 
non-superymmetric models such as these. It is conceivable too that radiative corrections could render the tree-level, charged-singlet tachyons nontachyonic. However, there is a further unwelcome feature of these models that seems unavoidable. It turns out that in the models under discussion there is one surviving additional $U(1)$ gauge symmetry, coupled to the observable matter, that is not spontaneously broken at the electroweak transition. We can do better if we abandon the requirement of mass terms for all matter at renormalisable level. Then there is a one-parameter family of six-stack models having stacks $1,2, i_{1}, j_{1}, j_{2}, k_{2}$ with no unwanted (coupled) surviving $U(1)$ gauge symmetry, but which has additional vector-like leptonic and quark matter. We find

$$
3\left(d_{L}^{c}+\bar{d}_{L}^{c}\right)+6(L+\bar{L})+6\left(e_{L}^{c}+\bar{e}_{L}^{c}\right)+3\left(\nu_{L}^{c}+\bar{\nu}_{L}^{c}\right)
$$

In addition there are 3 Higgs doublets, 3 charged-singet tachyonic scalars and 3 colour-triplet scalar tachyons.

We have also found two-parameter, eight-stack models having precisely one stack in each of the sets $I_{1}, I_{2}, J_{1}, J_{2}, K_{1}, K_{2}$, that have mass terms at renormalisable level for all matter and that are free of charged-singlet tachyons. As before, there is extra vector-like leptonic, but not quark, matter. The precise vector-like matter content depends upon one of the parameters. There are several values of this parameter that give gauge coupling constant ratios fairly close to those measured, and one value that comes very close. In this case, therefore, the string scale cannot be far from the electroweak scale. As before ${ }^{7}$, baryon number is anomalous, and survives as a global symmetry, so the proton is stable despite the low string scale. Unfortunately, these models too are afflicted with several unwanted $U(1)$ gauge symmetries that survive electroweak spontaneous symmetry breaking.

\section{D5-brane models}

It is natural to wonder whether similar models, constructed using D5branes that wrap $T^{2} \times T^{2}$ and are situated at a fixed point of $T^{2} / Z_{3}$, can improve upon the results of D4-brane models. In this case each stack $a$ is characterised by a total of four wrapping numbers $\left(n_{a}, m_{a}\right)\left(\tilde{n}_{a}, \tilde{m}_{a}\right)$, two for each wrapped torus. The intersection number is now given by $I_{a b}=\left(n_{a} m_{b}-m_{a} n_{b}\right)\left(\tilde{n}_{a} \tilde{m}_{b}-\tilde{m}_{a} \tilde{n}_{b}\right)$. Important differences from the D4brane case arise in connection with the tachyonic scalar states. As before, they arise at intersections of stacks having the same Chan-Paton factor; in fact, only scalar tachyons arise at such intersections in this case. The differ-

ence of the angles $\theta_{a b}$ and $\tilde{\theta}_{a b}$ between the intersecting 1-cycles on the two 
wrapped tori determine the squared mass of the tachyon at such an intersection, and when they are equal the tachyon is massless. Thus in D5-brane models potentially unwelcome (e.g. charged-singlet and/or colour-triplet) tachyons might be removable by this mechanism. A further difference is that tachyons may also arise when the intersecting D5-branes have parallel 1-cycles in one, but not both, of the two wrapped tori ${ }^{5}$, i.e. $\theta_{a b}=0$ or $\tilde{\theta}_{a b}=0$. The intersection number of the non-parallel 1-cycles determines the number of such states that arise. The distance between the parallel 1-cycles enters the mass formula, so the masses of these states are continuously adjustable. When it is zero (or small enough) the states are tachyonic, but when the cycles are far enough apart the states are massive and can be removed from the low-energy spectrum. There are eight constraints on the wrapping numbers deriving from twisted tadpole cancellation and requiring that the $U(1)_{Y}$ gauge boson associated with weak hypercharge does not get a Green-Schwarz mass. The constraints are quadratic in the wrapping numbers, and the only six-stack models that can satisfy them have stacks $1,2, i_{1}, i_{2}, k_{1}, k_{2}$. Unfortunately, the solution gives $I_{2 i_{1}}=0=I_{2 i_{2}}$ and, since the 1-cycles in both tori are parallel, these models have no Higgs doublets (or charged-singlet or colour-triplet tachyons). They are therefore not standard-like and we are forced to consider eight-stack models from the outset.

We may obtain non-zero intersection numbers for the Higgs doublets by taking the sets $I_{1}$ and $I_{2}$ to contain more than one stack. In particular, if we take each to have two stacks $i_{1}^{(1,2)}$ and $i_{2}^{(1,2)}$ respectively, then there are consistent eight-stack, three-generation models with just $j_{1}$ (or $k_{1}$ ) and $j_{2}$ (or $k_{2}$ ) stacks in addition. A priori, the Green-Schwarz mechanism gives string-scale masses to eight linear combinations of the $U(1)$ gauge fields. However, in the models we are considering three of these are lost, and the three surviving massless $U(1)$ gauge symmetries include (an unwanted) one which is coupled to observable matter, but which remains unbroken after electroweak spontaneous symmetry breaking. We have also investigated models in which the Higgs doublets arise from intersections on just one of the wrapped tori, the 1-cycles on the other being parallel. We find eightstack, three-generation models satisfying the constraints with just one stack in each of the sets $I_{1}, I_{2}, J_{1}, J_{2}, K_{1}, K_{2}$, and some of these are free of unwanted coupled $\mathrm{U}(1)$ gauge symmetries. The models have extra vector-like leptonic, but not quark, matter, and charged-singlet scalar tachyons. The two parameters may be chosen so that the tree-level gauge coupling constant ratios are equal to those measured, consistent with a string scale very close to the electroweak scale. These models will be analysed elsewhere ${ }^{14}$. 


\section{Acknowledgements}

It is a pleasure to acknowledge an enjoyable collaboration with George Kraniotis and Alex Love with whom all of this work was done. This account is a modified version of the talk I gave in Oxford in July, 2002. The research is supported in part by PPARC and by the German-Israeli Foundation for Scientific Research (GIF).

\section{References}

1. G. Aldazabal, L. E. Ibáñez and F. Quevedo, JHEP 0001(2000)031, hepth/9909172, JHEP 0002 (2000) 015, hep-th/0005067; I. Antoniadis, E. Kiritsis and T. Tomaras, Phys. Lett. B486 (2000) 186, hep-th/0004214; D. Bailin, G.V. Kraniotis, A. Love, Phys. Lett. B502 (2001) 209, hep-th/0011289; C. Bachas, hep-th/9503030; R. Blumenhagen, L. Görlich, B. Körs and D. Lüst, JHEP 0010 (2000) 006, hep-th/0007024; Z. Kakushadze, Phys. Rev. D59 (1999) 045007; Z. Kakushadze, G. Shiu and S.-H. Henry Tye, Nucl. Phys. B533(1998)25; J. Lykken, E. Poppitz, S. P. Trivedi, Nucl. Phys. B543 (1999) 105; I. Antoniadis, E. Dudas, A. Sagnotti, Phys. Lett.B 464 (1999) 38; S. Sugimoto, Prog. Theor. Phys. 102 (1999) 685; C. Angelantonj, Nucl. Phys.B $566(2000) 126$;

2. M. Berkooz, M. R. Douglas and R. G. Leigh, Nuclear Physics B480 (1996) 265, hep-th/9606139

3. N. Arkani-Hamed, S. Dimopoulos and G.R. Dvali, Phys. Lett. B429 (1998) 263, hep-ph/9803315; I. Antoniadis, N. Arkani-Hamed, S. Dimopoulos and G.R.Dvali, Phys. Lett.B 436 (1998) 257, hep-ph/9804398.

4. L. E. Ibáñez, F. Marchesano and R. Rabadan, JHEP 0111 (2001) 002, hepth/0105155

5. D. Cremades, L. E. Ibáñez and F. Marchesano, hep-th/0205074

6. R. Blumenhagen, V. Braun , B. Körs and D. Lüst, JHEP 0207 (2002) 026, hep-th/0206038 ; G. Aldazabal, L. E. Ibáñez, A.M. Uranga, hep-ph/0205250; G. Honecker, JHEP 0201 (2002) 025, hep-th/0201037, hep-th/0112174; H. Kataoka and M. Shimojo, hep-th/0112247; C. Kokorelis, hep-th/0203187, hepth/0205147; J. R. Ellis, P. Kanti, D.V. Nanopoulos, hep-th/0206087

7. D. Bailin, G. V. Kraniotis and A. Love, Physics Letters B530 (2002) 202, hep-th/0108131

8. L. E. Ibáñez, R. Rabadán and A. M. Uranga, Nucl. Phys. B542 (1999) 112, hep-th/9808139

9. G. Aldazabal, S. Franco, L. E. Ibáñez, R. Rabadán and A. M. Uranga, JHEP 0102 (2001) 047, hep-ph/0011132

10. D. Bailin, G. V. Kraniotis and A. Love, hep-th/0208103

11. D. M. Ghilencia and H. P. Nilles, hep-th/0204261

12. Reports of the working groups on precision calculations for LEP2 Physics, S. Jadach, G. Passarino, R. Pittau (Eds.), CERN Yellow Report, CERN, 2000009

13. D. M. Ghilencia, L. E. Ibáñez, N. Irges and F. Quevedo, hep-ph/0205083

14. D. Bailin, G. V. Kraniotis and A. Love, in preparation 